\title{
Verstehen, Interesse und Fachsprache im Physikunterricht
}

\author{
Mag. . Maria Stampfl \\ Institut für Astro- und Teilchenphysik \\ Universität Innsbruck \\ ao. Univ.-Prof. Dr. Walter Saurer \\ Institut für Astro- und Teilchenphysik \\ Universität Innsbruck
}

Ein wesentliches Ziel des Physikunterrichts besteht darin, dass die SchülerInnen die Fachsprache verstehen und anwenden können. Viele SchülerInnen haben jedoch Probleme mit der Erreichung dieses Ziels. Die physikalische Fachsprache wird nach Leisen (2010) gern in Merksätzen und Definitionen verwendet. Sie ist durch eine hohe Dichte an Fachbegriffen sowie durch Satz- und Textkonstruktionen gekennzeichnet, die in der Allgemeinsprache selten vorkommen (z.B. Fachbegriffe wie „Auftriebskraft", „Schweredruck“, „eine Kraft erfahren“ oder Sätze wie „Taucht ein ... in ... ein, so wird ...").

Als Merkmal der physikalischen Fachsprache stellt Muckenfuß (1995: 259) fest: „Physikalische Fachbegriffe sind ,theoriegeladen‘: Jeder Begriff ist Element eines ganzen Begriffsnetzes, dem eine physikalische Theorie zugrunde liegt". Nach Merzyn (2008) treten im naturwissenschaftlichen Unterricht in einer Unterrichtsstunde etwa neun neue Fachbegriffe auf. Naturwissenschaftliche Schulbücher der Sekundarstufe I enthalten etwa 1500 bis 2500 verschiedene Fachbegriffe. Rund 50\% aller Fachbegriffe werden im Lehrwerk jedoch nur ein einziges Mal benutzt. Ein wesentliches Problem der Fachsprache im Physikunterricht stellen aus der Alltagssprache übernommene Fachbegriffe dar, die teilweise mit einer neuen Bedeutung versehen werden. Ein sprachsensibler Fachunterricht in Physik sollte deshalb die bereits vorhandenen sprachlichen und fachlichen Kompeten-

Barbara Hinger (Hg.), Zweite „Tagung der Fachdidaktik“ 2015.

Sprachsensibler Sach-Fach-Unterricht - Sprachen im Sprachunterricht.

(C) 2016 innsbruck university press, ISBN 978-3-903122-51-2, DOI 10.15203/3122-51-2 
zen der SchülerInnen berücksichtigen. Dabei ist es wesentlich, auf die bereits vorhandenen SchülerInnenvorstellungen in Bezug auf das Fach Physik, sogenannte Präkonzepte, einzugehen (Duit 2003).

Erste Ergebnisse aus einem aktuellen, in den folgenden Ausführungen beschriebenen Forschungsprojekt weisen auf einen bedeutenden Zusammenhang zwischen der Interessensentwicklung der SchülerInnen am Physikunterricht und den Schwierigkeiten hin, die sie mit der Fachsprache haben. Studien zur Interessensentwicklung im Physikunterricht stimmen mit Befunden zahlreicher Forschungsarbeiten (Hoffmann et al. 1998; Höner 1996; Todt 1993) aus der Fachdidaktik der Naturwissenschaften überein, welche den Rückgang des Interesses am Physikunterricht insbesondere bei Mädchen belegen (Kessels et al. 2006; Wodzinski 2007).

\section{Ziel und Fragestellungen des Forschungsprojektes}

Ziel des Forschungsprojektes ist die Erhebung der derzeitigen Interessensentwicklung am Physikunterricht von SchülerInnen der Sekundarstufe I und die Identifikation möglicher Prädiktoren, die eine positive oder negative Interessensentwicklung begünstigen. Theoretische Grundlagen sind dabei die Interessentheorie von Krapp (1998, 2002, 2005) sowie von Deci \& Ryan (1993). Ausgehend von dieser Zielsetzung und den Grundlagen der pädagogisch-psychologischen Interessensforschung ergeben sich die folgenden Forschungsfragen:

- Wie groß ist das Interesse der SchülerInnen am Physikunterricht? Bestehen geschlechtsspezifische Ausprägungen?

- Wie hat sich das Interesse der SchülerInnen am Physikunterricht im Laufe der Sekundarstufe I verändert?

- Welche Faktoren sind für die SchülerInnen für ihr Interesse bzw. ihre Interessensentwicklung am Physikunterricht bestimmend? 


\section{Operationalisierung und Untersuchungsinstrumente}

Die Studie umfasst eine Quer- und eine Längsschnittuntersuchung und nutzt zur Datenauswertung eine Kombination von qualitativen und quantitativen Untersuchungsmethoden unter Anwendung des Konzeptes der Triangulation (Flick 2004). Im Rahmen der Querschnittuntersuchung wurde den ProbandInnen aller vier Schulstufen der Sekundarstufe I ein Fragebogen vorgelegt. Die SchülerInnen der ersten Schulstufe der Querschnittuntersuchung wurden als TeilnehmerInnen der vierjährigen Längsschnittstudie in den folgenden Schuljahren anhand eines Fragebogens zu ihrem Interesse am Physikunterricht befragt. Zudem wurden fünfunddreißig SchülerInnen in jedem Schuljahr interviewt, von denen zwei davon nicht an allen Befragungszeitpunkten interviewt werden konnten.

Der Fragebogen wurde theoriebasiert erstellt, wobei auf bewährte Befragungsinstrumente aus der Fachdidaktik zurückgegriffen wurde. Er enthält allgemeine Fragen zu Herkunft, Alter und Geschlecht der SchülerInnen sowie Fragen zu den sogenannten „basic needs“ (Krapp 2005), zum Kompetenzerleben, zur sozialen Eingebundenheit und zum Autonomieerleben. Die Skala des individuellen Fachinteresses an Physik besteht aus sieben Items (Müller et al. 2007) und beinhaltet, wie in der Interessenstheorie postuliert, eine wertbezogene, eine gefühlsbezogene und eine epistemische Komponente. Weitere mögliche Prädiktoren für das Interesse am Physikunterricht, die erfasst wurden, sind: Das fachspezifische Fähigkeitsselbstkonzept, die Motivierungsqualität und Instruktionsqualität der Lehrperson, die persönliche Bedeutsamkeit, Merkmale der Unterrichtsqualität und die Relevanz der Inhalte des erlebten Physikunterrichts (Hoffman et al. 1998; Müller \& Thomas 2011; Priemer 2003; Waldis et al. 2010).

Für die Auswertung der Fragebögen der Quer- und Längsschnittstudie wurden deskriptive und inferenzstatistische Methoden angewandt. Die statistische Analyse der Daten erfolgte mit dem Statistikprogramm SPSS. Für die Auswertung von Mittelwertsunterschieden wurde der Mann-Whitney-U-Test angewendet, für die Zusammenhangshypothesen die logistische Regression GLM.

Um einerseits ausreichend vergleichen zu können und andererseits auch Spielraum für offene Antworten zu geben, wurden qualitative Erhebungen in Form von halbstandardisierten Interviews mit fünfunddreißig zufällig ausgewählten

Barbara Hinger (Hg.), Zweite „Tagung der Fachdidaktik“ 2015.

Sprachsensibler Sach-Fach-Unterricht - Sprachen im Sprachunterricht.

(C) 2016 innsbruck university press, ISBN 978-3-903122-51-2, DOI 10.15203/3122-51-2 
ProbandInnen durchgeführt. Diese erstreckten sich über vier Jahre mit einem Befragungszeitpunkt in der Mitte des Schuljahres. Die SchülerInnen der ersten Klasse, die laut Lehrplan noch keinen Physikunterricht hatten, wurden am Ende des ersten Schuljahres zu ihrer Einstellung und ihrem Interesse am zukünftigen Physikunterricht befragt.

Die Interviews wurden mit dem Programm f4 transkribiert und im Softwareprogramm MAXQDA codiert. Für die Analyse wurden die Regeln der qualitativen Inhaltsanalyse nach Mayring (2010) angewandt, die formal an die Software MAXQDA (Kuckartz 2010) angepasst wurden. Einen Überblick über die Inhaltsanalyse bietet Abb. 1.

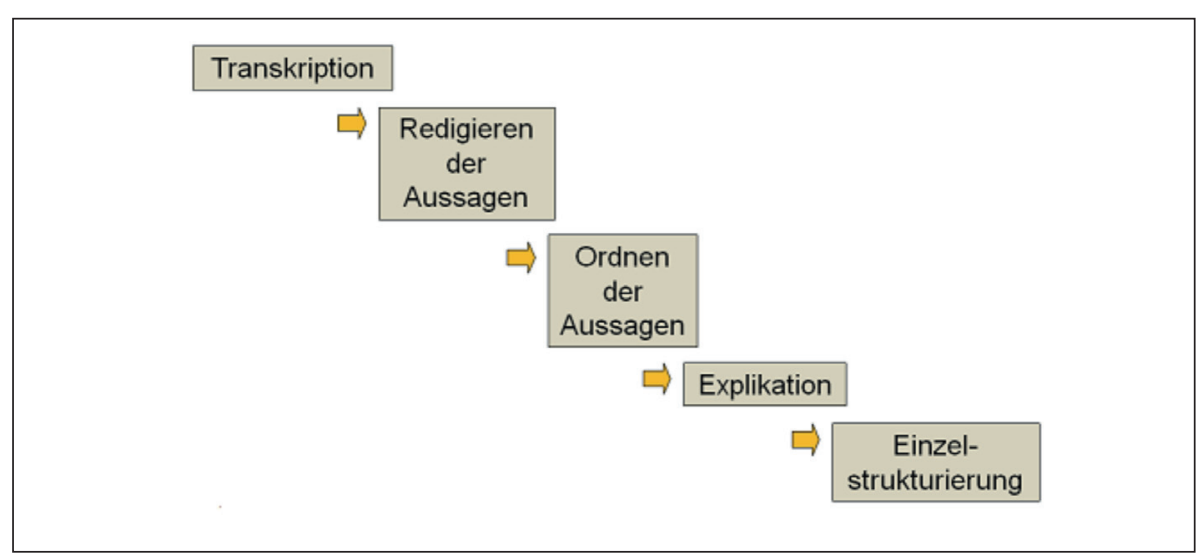

Abb. 1: Überblick über die Inhaltsanalyse

Erstes Ziel der Auswertung ist die Strukturierung eines Kategoriensystems. Als theoretische Grundlage für die Erfassung kategorienbildender Merkmale dienten die folgenden acht interessensunterstützenden Unterrichtsbedingungen: Die drei psychologischen Grundbedürfnisse Autonomie, Kompetenz und soziale Eingebundenheit (Krapp 2005), das fachspezifische Fähigkeitsselbstkonzept (Duit 1997), die Merkmale der Unterrichtsqualität, die Relevanz der Inhalte und die Instruktions- bzw. Motivierungsqualität der Lehrperson (Prenzel et al. 2001; Waldis 2012). 


\section{Ergebnisse}

Die im Folgenden angeführten Ergebnisse beziehen Auswertungen jener Interviews ein, die in den ersten drei Klassen geführt wurden. Die Resultate der Interviews der vierten Klasse sowie jene der Fragebogenerhebungen sind aktuell in Bearbeitung und werden in weiteren Publikationen vorgestellt.

Aufgrund der SchülerInnenaussagen in den Interviews zeigt sich zunächst, dass der Frontalunterricht im Durchschnitt immer noch ca. 90\% der Unterrichtszeit ausmacht. Jede Physikstunde beginnt mit einer ca. zehnminütigen Stundenwiederholung in Form einer mündlichen Prüfung eines bzw. mehrerer SchülerInnen an der Tafel. Anschließend wird das neue Thema der jeweiligen Unterrichtseinheit frontal den Lernenden vermittelt. Dazu werden Texte diktiert oder an die Tafel geschrieben. Manchmal führt die Lehrperson einen Demonstrationsversuch durch. SchülerInnenversuche finden in der Regel sehr selten durchschnittlich zweimal im Jahr - statt. Der Laborunterricht ist nach Aussage der SchülerInnen eine sehr gute, positive Unterstützung des Regelunterrichts. Die SchülerInnen erarbeiten sich dabei nicht nur die Theorie physikalischer Phänomene, sondern können erkennen und erfahren, dass das Besprochene auch in der Praxis funktioniert.

Bezogen auf die oben dargelegten Forschungsfragen zeigt Abb. 2 eine vorläufige Auswertung der Interviews hinsichtlich der Interessensentwicklung am Physikunterricht im Laufe der Sekundarstufe. Abb. 2 zeigt die Resultate getrennt nach Geschlechtern. Gesamt ist festzustellen, dass dreizehn Schülerinnen und vier Schüler kein Interesse am Physikunterricht haben. Zwei Schülerinnen und acht Schüler zeigen ein durchgehendes Interesse am Physikunterricht. Bei zwei Schülerinnen respektive vier Schülern ist das Interesse am Physikunterricht im Laufe des Untersuchungszeitraums gestiegen. Die Geschlechterdifferenz verweist darauf, dass mehr Schüler als Schülerinnen durchgehend Interesse am Physikunterricht haben (8 Schüler gegenüber zwei Schülerinnen), deutlich weniger Schüler (4) als Schülerinnen (13) kein Interesse am Physikunterricht haben und geringfügig mehr Schüler (4) ein steigendes Interesse am Physikunterricht zeigen als Schülerinnen (2).

Barbara Hinger (Hg.), Zweite „Tagung der Fachdidaktik“ 2015.

Sprachsensibler Sach-Fach-Unterricht - Sprachen im Sprachunterricht.

(C) 2016 innsbruck university press, ISBN 978-3-903122-51-2, DOI 10.15203/3122-51-2 


\begin{tabular}{|l|c|c|}
\hline & Schülerinnen N=17 & Schüler N=16 \\
\hline Jedes Jahr Interesse am PU & 2 & 8 \\
\hline Kein Interesse am PU & 13 & 4 \\
\hline Interesse am PU gestiegen & 2 & 4 \\
\hline
\end{tabular}

Abb. 2: Entwicklung des Fachinteresses an Physik im Laufe der Sekundarstufe I (Anzahl der SchülerInnen N = 33)

Vergleichbare Ergebnisse zur Interessensentwicklung zeigen sich auch in Studien zu den Schulstufen fünf bis zehn (Krapp 1998). Darüber hinaus ist feststellbar, dass sich SchülerInnen der ersten Klasse der Sekundarstufe I in der Regel auf den zukünftigen Physikunterricht freuen, der für sie üblicherweise in der zweiten Klasse einsetzt. (Bereits) nach den ersten Monaten des Physikunterrichts zeigt sich jedoch insbesondere bei Schülerinnen ein geringeres Interesse am Physikunterricht. Dieses Ergebnis bestätigt in der Fachliteratur bereits vorgestellte Resultate (z. B. Häußler \& Hoffmann 1998).

Für den Interessensabfall im Physikunterricht gibt es unterschiedliche Gründe. Auffallend ist, dass insbesondere Schülerinnen die Inhalte des Physikunterrichts langweilig und uninteressant finden. So äußert sich etwa eine Schülerin in einem der Interviews wie folgt: „Man könnte die Sachen und die Themen und so, ein bisschen anders rüberbringen als unser Physiklehrer, weil der schreibt ein Thema an die Tafel und sagt das ist das, und es interessiert eigentlich niemanden. Weil das einfach uninteressant ist. Er erzäblt das schon uninteressant und manchmal kommt mir vor, der Lehrer schläft selbst dabei ein".

Darüber hinaus geht aus der Befragung hervor, dass sich einige - insbesondere weibliche - SchülerInnen, nicht begabt für den Physikunterricht fühlen und dies als einen wesentlichen Grund für das fehlende Interesse am Unterrichtsfach angeben. Ein ebensolches Ergebnis findet sich auch in Duit (1997: 7): „Eine wichtige Rolle spielt vielmehr, ob sich die SchülerInnen das als schwierig erachtete Fach zutrauen, also das Vertrauen in die eigene Fähigkeit“". 
Die SchülerInnen finden außerdem, dass es für sie sehr wichtig ist, ob sie etwas gut können oder nicht, und dass mangelnde Kompetenz ein wesentlicher Grund für das Desinteresse im Physikunterricht darstellt. Daraus kann geschlossen werden, dass SchülerInnen das Interesse verlieren, wenn sie den Unterricht nicht (gut) verstehen oder dessen Inhalte nicht anwenden können. Auch nach Krapp (2002) spielt das Kompetenzerleben eine wichtige Rolle für die Entwicklung und den Erhalt von Interesse im Unterricht. Die dafür ausschlaggebenden Gründe sehen die Lernenden vorwiegend in den Erklärungen der Lehrperson, also in deren Instruktionsqualität, aber auch in der fehlenden Aufmerksamkeit ihrerseits. Den Unterricht im Allgemeinen empfinden viele SchülerInnen als zu schwierig, zu kompliziert und zu wenig anschaulich. Die Lehrperson erkläre zu wenig, zu schnell, zu ungenau oder zu kompliziert. Bei Rückfragen würden dieselben Erklärungen oft wiederholt.

Schwierigkeiten in Bezug auf die Instruktion betreffen vor allem den Sprachgebrauch im Physikunterricht, also die Fachsprache, die verwendeten Abkürzungen, aber auch ganze Sätze und verständliche Formulierungen. Dabei bereiten den SchülerInnen Fachbegriffe wie die Teile eines Elektromotors (z. B. Stator, Rotor usw.) geringe Schwierigkeiten. Diese Begriffe lernen sie auswendig. Die physikalische Bedeutung anderer aus dem Alltagsleben bekannter Begriffe wie Kraft, Arbeit, Energie usw. ist für SchülerInnen jedoch komplizierter. Eine weitere Schwierigkeit ergibt sich aus der Tatsache, dass manche Begriffe mit einer doppelten Bedeutung (elektrische Spannung, Federspannung) versehen sind oder für mehrere Bezeichnungen dieselbe Abkürzung verwendet wird (m für Masse und für Meter, W für Arbeit und für Watt). Wenn SchülerInnen einen Text aus dem Physikbuch mit eigenen Worten zusammenfassen sollen, haben sie oft Schwierigkeiten, die Fachsprache zu verstehen. Der Text ist für sie zu kompliziert verfasst und sie sind sich nicht sicher, ob sie das Wichtigste notiert oder zu viel Unwichtiges aufgeschrieben haben.

Die Einstellungen in Bezug auf das Interesse am Physikunterricht haben sich bei den SchülerInnen bereits in der dritten Klasse der Sekundarstufe I gefestigt. Besonders die Schülerinnen fühlen sich nicht ausreichend begabt für den Physikunterricht. Sie geben an, die im Physikunterricht gelernten Inhalte im weiteren Leben nicht mehr zu benötigen, weshalb ihnen diese Inhalte nicht wichtig sind.

Barbara Hinger (Hg.), Zweite „Tagung der Fachdidaktik“ 2015.

Sprachsensibler Sach-Fach-Unterricht - Sprachen im Sprachunterricht.

(C) 2016 innsbruck university press, ISBN 978-3-903122-51-2, DOI 10.15203/3122-51-2 
Auch wenn die SchülerInnen an der Art des Physikunterrichts wenig bis nichts auszusetzen haben, bleibt das Desinteresse am Physikunterricht bei den meisten bestehen.

Die Schüler, deren Interesse im Laufe der Sekundarstufe I gestiegen ist, geben an, dass dieses Fach auch in der weiterführenden Schule, die sie besuchen möchten, unterrichtet wird, oder, weil sie einen guten Notendurchschnitt für die weiterführende Schule benötigen. Aus diesen Gründen ist ihr Lerninteresse für den Physikunterricht höher, wodurch sie die Inhalte besser kennen, mehr von den Themen verstehen und insgesamt mehr Interesse am Physikunterricht haben. Jene beiden Schülerinnen, die angeben, dass ihr Interesse am Physikunterricht im Laufe der Jahre gestiegen ist, betonen, dass sie den Unterricht besser verstehen und bessere Noten bekommen haben. Aus diesen Gründen ist ihr Interesse am Physikunterricht gestiegen.

\section{Konklusion und Ausblick}

Insgesamt zeigt die Auswertung der Interviews, dass SchülerInnen, die über zumindest ein mittleres Fähigkeitsselbstkonzept verfügen, sich im Physikunterricht kompetent fühlen und dessen Inhalte verstehen. Dass das Zutrauen in die eigenen Fähigkeiten (Fähigkeitsselbstkonzept) einen Einfluss auf das Kompetenzerleben und im weiteren auf das Fachinteresse hat, wird in mehreren Studien (Daniels 2008; Deci \& Ryan 2008) bestätigt. Trotzdem ist das Interesse am Physikunterricht vor allem bei den Mädchen nicht sehr ausgeprägt, da sie an den gebotenen physikalischen Themen wenig Relevanz sehen. Bestätigt werden diese Ergebnisse auch von SchülerInnen, die an der PISA-Studie teilgenommen haben und angeben, dass die Unterrichtsinhalte in Physik nicht nur komplex und schwer fassbar, sondern schlicht nicht interessant genug sind (vgl. Prenzel 2005). Eine stärkere Anbindung an die Lebenswelt der SchülerInnen (Kontextualisierung) wird in der IPN-Studie und der ROSE-Studie (Sjøberg \& Schreiner 2010) gefordert. Der Physikunterricht wird dann besonders interessant für SchülerInnen, wenn sie eigene Experimente durchführen können (Logan \& Skamp 2008; Möller 2014). Auch die interviewten SchülerInnen

Barbara Hinger (Hg.), Zweite „Tagung der Fachdidaktik“ 2015.

Sprachsensibler Sach-Fach-Unterricht - Sprachen im Sprachunterricht.

(C) 2016 innsbruck university press, ISBN 978-3-903122-51-2, DOI 10.15203/3122-51-2 
wünschen sich mehr Experimente, wobei zu bedenken ist, dass das Experimentieren ohne eine gute Vor- und Nachbereitung nicht per se zu einem höheren individuellen Fachinteresse führt (Duit et al. 2010). Darüber hinaus ist das Fach Physik trotz guten Unterrichts und guter Erklärungen für die SchülerInnen schwieriger als andere Fächer. Deshalb würden einige SchülerInnen ohne weiteres auch auf den Physikunterricht verzichten.

Abschließend ist auf folgende Verbesserungsvorschläge für den Physikunterricht zu verweisen, die von den SchülerInnen angeführt werden:

- SchülerInnen wünschen sich mehr Mitspracherecht bei Kontexten und Inhalten.

- Sie wünschen sich unterschiedliche Unterrichtsmethoden, Gruppen- und Partnerarbeiten sowie eigenständiges Arbeiten.

- Für den Laborunterricht zeigen auch schwächere SchülerInnen Interesse, da sie dort selbst etwas aufbauen und erarbeiten können. Im üblichen Physikunterricht wird demgegenüber die Autonomie oder Selbstbestimmung der SchülerInnen in der Regel nicht oder wenig unterstützt. Deshalb finden die SchülerInnen, dass der Laborunterricht beibehalten und deutlich verstärkt werden sollte.

- In einer Unterrichtsstunde sollten nur einzelne neue Fachbegriffe vorkommen.

- Die Fachbegriffe sollten in der Stunde mehrmals wiederholt werden; deren Bedeutung sollte im Heft vermerkt werden.

- Die Lehrperson sollte gut erklären, genau erklären, auf unterschiedliche Art und Weise erklären und jedenfalls mehr Erklärungen geben.

- Die Lehrperson sollte durch Nachfragen klären, ob die SchülerInnen neue Inhalte verstanden haben.

- Bei Abkürzungen sollte immer auch notiert werden, was sie bedeuten.

- Während des Frontalunterrichts sollte zuerst erklärt und erst danach aufgeschrieben werden. Beides zugleich erscheint den SchülerInnen als zu komplex. Es sollte das aufgeschrieben werden, was wichtig ist, damit die SchülerInnen die Inhalte ein paar Wochen später auch entsprechend nachvollziehen können. 
- Die Lehrperson sollte beim Aufschreiben von komplexeren Inhalten, über die auch reflektiert werden muss, den SchülerInnen Zeit zum Nachdenken geben und dürfte nicht zu schnell zu weiteren Inhalten übergehen.

- Die SchülerInnen wünschen sich mehr vorgeführte Experimente und vor allem mehr Experimente, die sie selbst durchführen können.

Weitere Aufschlüsse über mögliche Prädiktoren für das Fachinteresse sind aus den Ergebnissen der Interviews der vierten Klasse und den Ergebnissen der Fragebogenerhebung zu erwarten, die in Folgepublikationen vorgestellt werden.

Das Projekt wurde vom Land Tirol durch den Wissenschaftsfond des Landes Tirol finanziell unterstützt (Projekt UNI - 0404/935).

\section{Literatur}

Daniels, Z. (2008). Entwicklung schulischer Interessen im Jugendalter. Münster: Waxmann.

Deci, E. L. \& Ryan, R. M. (1993). Die Selbstbestimmungstheorie der Motivation und ihre Bedeutung für die Pädagogik. Zeitschrift für Pädagogik, 39 (2), 223-238.

Deci, E. L. \& Ryan, R. M. (2008). Self-Determination Theory: A Macrotheory of Human Motivation, Development, and Health. Canadian Psychology, 49, 182-185.

Duit, R. (1997). Ziele für den naturwissenschaftlichen Unterricht - Anspruch und Realität. Plus Lucis, 1, 3-13.

Duit, R. (2003). Alltagsvorstellungen und Physik lernen. In E. Kircher \& W. B. Schneider (Hrsg.), Physikdidaktik in der Praxis (1-26). Berlin: Springer.

Duit, R., Tesch, M. \& Mikelskis-Seifert, S. (2010). Das Experiment im Physikunterricht. Piko-Brief Nr. 7. Verfügbar unter: www.ipn.uni-kiel.de/de/das-ipn/abteilungen/didaktik-der-physik/piko/ pikobriefe032010.pdf [29.03.2016].

Flick, U. (2004). Triangulation. Eine Einführung. Wiesbaden: VS Verlag für Sozialwissenschaften.

Häußler, P. \& Hoffmann, L. (1998). Chancengleichheit für Mädchen im Physikunterricht - Ergebnisse eines erweiterten BLK-Modellversuchs. Zeitschrift für Didaktik der Naturwissenschaften, 4 (1), 51-67.

Barbara Hinger (Hg.), Zweite „Tagung der Fachdidaktik“ 2015.

Sprachsensibler Sach-Fach-Unterricht - Sprachen im Sprachunterricht.

(C) 2016 innsbruck university press, ISBN 978-3-903122-51-2, DOI 10.15203/3122-51-2 
Hoffmann, L., Häußler, P. \& Lehrke, M. (1998). Die IPN - Interessenstudie. Universität Kiel.

Höner, K. (1996). Mathematisierung im Chemieunterricht - ein Motivationshemmnis? Zeitschrift für Didaktik. der Naturwissenschaft, 2 (2), 51-70.

Kessels, U., Rau, M. \& Hannover, B. (2006). What goes well with physics? Measuring and altering the image of science. British Journal of Educational Psychology, 76, 761-780.

Krapp, A. (1998). Entwicklung und Förderung von Interessen im Unterricht. Psychologie in Er:iebung und Unterricht, 44, 185-201.

Krapp, A. (2002). An educational-psychological theory of interest and its relation to self determination theory. In E. L. Deci \& R. M. Ryan (Hrsg.), The bandbook of self determination research (405-427). Rochester: University of Rochester Press.

Krapp, A. (2005). Basic needs and the development of interest and intrinsic motivational orientations. Learning and Instruction, 15, 391-395.

Kuckartz, U. (2010). Einführung in die computergestütste Analyse qualitativer Daten. Wiesbaden: VS Verlag für Sozialwissenschaften.

Leisen, J. (2010). Handbuch Sprachförderung im Fach - Sprachsensibler Fachunterricht in der Praxis. Bonn: Varus.

Logan, M. \& Skamp, K. (2008). Engaging Students in Science across the Primary Secondary Interface: Listening to the Students`Voice. Research in Science Education, 38, 501-527.

Mayring, P. (2010). Qualitative Inhaltsanalyse: Grundlagen und Techniken. Weinheim: Beltz.

Merzyn, G. (2008). Naturwissenschaften Mathematik Technik - immer unbeliebter? Baltmannsweiler: Schneider Verlag Hohengehren.

Möller, K. (2014). Vom naturwissenschaftlichen Sachunterricht zum Fachunterricht - Der Übergang von der Grundschule in die weiterührende Schule. Zeitschrift für Didaktik der Naturwissenschaften, 20, 33-43.

Müller, F., Hanfstingl, B. \& Andreitz, I. (2007). Skalen zur motivationalen Regulation beim Lernen von Scbülerinnen und Scbülern. Adaptierte und ergänzte Version des Academic Self-Regulation Questionnaire (SRQ-A) nach Ryan \& Connell. Wissenschaftlicher Bericht Nr. 1. Alpen-Adria-Universität Klagenfurt.

Müller, F. \& Thomas, A. (2011). Skalen zur wabrgenommenen Basic Needs Unterstützung von Schüler/ innen. Alpen-Adria-Universität Klagenfurt.

Muckenfuß, H. (1995). Lernen im sinnstiftenden Kontext. Entwurf einer zeitgemäßen Didaktik des Physikunterrichts. Berlin: Cornelsen.

Barbara Hinger (Hg.), Zweite „Tagung der Fachdidaktik“ 2015.

Sprachsensibler Sach-Fach-Unterricht - Sprachen im Sprachunterricht.

(C) 2016 innsbruck university press, ISBN 978-3-903122-51-2, DOI 10.15203/3122-51-2 
Prenzel, M. (2005). PIS A 2003: Der zweite Vergleich der Länder in Deutschland - was wissen und können Jugendliche? Münster, München: Waxmann.

Prenzel, M., Drechsel, B. \& Kramer, K. (2001). Self-determined and interested learning in vocational education. In K. Beck (Hrsg.), Teaching-learning process in initial business education (43-68). Boston: Kluwer.

Priemer, B. (2003). Ein diagnostischer Test zu Schüleransichten über Physik und Lernen von Physik - eine deutsche Version des Tests „Views About Science Survey”. Zeitschrift für Didaktik der Naturwissenschaften, 9, 160-178.

Sjøberg, S. \& Schreiner, C. (2010). The ROSE project. An overview and key findings. Verfügbar unter: http://roseproject.no/network/countries/norway/eng/nor-Sjoberg-Schreiner-overview2010.pdf [29.03.2016].

Todt, E. (1993). Schülerempfehlungen für einen interessanten Physikunterricht. Naturwissenschaften im Unterricht-Physik, 4 (18), 37-40.

Waldis, M. (2012). Interesse an Mathematik. Zum Einfluss des Unterrichts auf das Interesse von Scbülerinnen und Scbülern der Sekundarstufe I. Münster: Waxmann.

Waldis, M., Grob, U., Pauli, C. \& Reusser, K. (2010). Der Einfluss der Unterrichtsgestaltung auf Fachinteresse und Mathematikleistung. In K. Reusser, C. Pauli \& M. Waldis (Hrsg.), Unterrichtsgestaltung und Unterrichtsqualität - Ergebnisse einer internationalen und schweizerischen Videostudie zum Mathematikunterricht (209-251). Münster: Waxmann.

Wodzinski, R. (2007). Mädchen im Physikunterricht. In E. Kircher, R. Girwidz \& P. Häußler (Hrsg.), Physikdidaktik Theorie und Praxis (559-580). Berlin: Springer. 